\title{
Rheological characterisation of gluten from extensibility measurement
}

\begin{abstract}
A simple tensile test set-up was built and attached to an Instron 5566 to determine gluten extensibility, comparing the performance of strong and weak flour mixed for various mixing time. In this work, the gluten strip was clamped at two ends and extended upward at the centre by a hook at speed of $300 \mathrm{~mm}$ min-1. Parameters such as gluten length at fracture, measured force, actual force acting on the gluten strips, strain, strain rate and stress were obtained. Gluten obtained from strong flour has greater extensibility compared to weak flour. The extensibility of gluten from both strong and weak flour dough increased as dough mixing time increased before decreasing at a peak of $8 \mathrm{~min}$. The results demonstrated the capability of the tensile test set-up to describe the development of gluten during mixing of dough. Rheological characteristic of gluten indicated that gluten exhibited strain hardening effect during extension.
\end{abstract}

Keyword: Curve-fitting, Extensibility, Gluten, Strain hardening, Tensile test 